\title{
EVALUATING GROUP-BASED RELEVANCE FEEDBACK FOR CONTENT-BASED IMAGE RETRIEVAL
}

\author{
Munehiro Nakazato, Charlie Dagli and Thomas S. Huang \\ Beckman Institute for Advanced Science and Technology \\ University of Illinois at Urbana-Champaign \\ 405 North Mathews Ave. Urbana, IL 61801 USA \\ \{nakazato, dagli, huang\}@ifp.uiuc.edu
}

\begin{abstract}
ABSTRAC'T
We have been developing new relevance feedback algorithms for Content-based Image Retrieval (CBIR) that allow the user to achieve more flexible query. In conjunction with the new user interface, called grouporiented user interface, the user's interest can be expressed with multiple groups of positive and negative image examples. This provides the users with greater flexibility compared with the previous systems where image query is considered as one-class or two-class problems.

In this paper, we analyze our new algorithm qualitatively and quantitatively. For comparison with the previous approaches, the systems are tested on both toy problems and real image retrieval tasks. From the result of our experiments, we suggest when and how our algorithm has advantages over the previous methods.
\end{abstract}

\section{INTRODUCTION}

In Content-Based Image Retrieval (CBIR) systems, Queryby-Example (QbE) method is commonly used [2][5]. In $\mathrm{QbE}$, the users are asked to select relevant images as samples. The system then retrieves similar images from the database. Often, it is also possible to specify irrelevant images as negative examples [6][7]. In these systems, image retrieval is considered as two-class (positive and negative) problems.

However, the user's high level concepts are often hard to be expressed with only two classes of image examples. For example, assume a user is looking for images of "cars." The query examples may contain various colors of cars. While images of "red cars" and "silver cars" have common features, they differ in terms of color features. In the traditional systems, these images are considered as one group of positive examples and everything else is assumed irrelevant. Therefore, much color features have to be ignored for the retrieval. These color features however, may be still valuable to discriminate images of "cars" from other images.

In [3], Nakazato et al. proposed a new graphical user interface for CBIR named ImageGrouper (Figure 1.) ImageGroper provides the user with a way to specify his/ her interest with multiple positive and multiple negative image groups. In this system, the users simply create image groups by drawing rectangles on the workspace and dragging images into the rectangles. For the example of "cars," the user can create two positive groups by separating red cars from silver cars. Other images are classified into a group of negative examples (Figure 1.) This query method is called Query-by-Groups [3].

In order to take advantage of this user interface for advanced query, relevance feedback algorithm [5][6][7] need to be extended. Thus, the new relevance feedback algorithm is proposed in [4]. In the new algorithm, Group Biased Discriminant Analysis (GBDA), handles image retrieval problems as classification of multiple positive classes and multiple negative classes. While the concept and algorithm of GBDA were clearly presented in [4], the system has not been evaluated in detail yet.

In this paper, we analyze the Group Biased Discriminant Analysis algorithm qualitatively and quantitatively. For comparison with the previous methods, the systems are tested on both low-dimensional toy problems and highdimensional real image retrieval tasks. From the analysis of the system, we suggest when our algorithm has advantages and how the system should be used in the CBIR domain.

The rest of paper is organized as follows. In the next section, we present the related work on relevance feedback

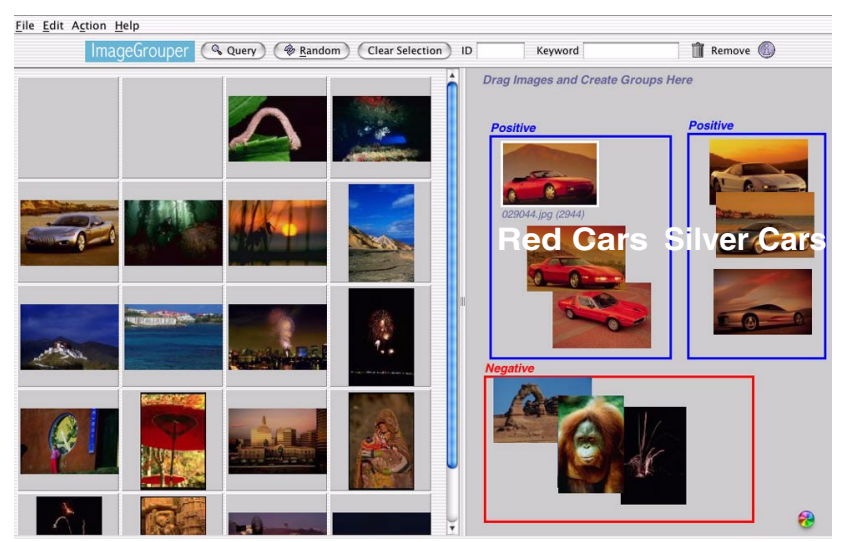

Figure 1. ImageGrouper, the user interface for flexible CBIR. In this example, the user created two positive groups and one negative group on the right workspace. (see [3] for more detail) 
for CBIR. In Section 3. the Group Biased Discriminant Analysis is briefly described. In Section 4. we analyze the effect of our algorithm on toy problems of three dimensional data. In Section 5. we evaluate the performance of the algorithm on real image retrieval tasks. The conclusion is presented in Section 6.

\section{RELATED WORK}

\subsection{Image retrieval as one and two class problems}

The most straightforward form of image retrieval systems simply asks the user to specify one or more relevant images. To improve the query results, some systems allow the user to manually change the weight of image features [2]. In MARS [5], the weights are automatically adjusted by relevance feedback where the user can repeatedly refine the query by giving feedback to the previous query result. The weights are adjusted by calculating the variance of each feature among the query examples. Then, the inverse of variance becomes the weight of the feature [5]. This gives higher weights to features in which example images are similar and gives lower weights to that features where the images differ.

Next, some systems allow the users to specify irrelevant images as negative examples [6][7]. Now the problem becomes two-class (positive and negative) problems. One solution is to use the Fisher's discriminant analysis (FDA) [1]. This approach, however, introduces undesirable side effects because it tries to cluster negative examples into one class. In the actual scenario, negative examples can be any types of images in the database.

\subsection{Image retrieval as a one to many class problem}

To address above problems, Zhou et al. [7] proposed a new relevance feedback algorithm, named Biased Discriminant Analysis (BDA), which effectively manges examples. They consider the relevance feedback problem as $(1+x)$-class (one positive and multiple negative classes) problem. In this algorithm, it is assumed the negative examples are coming from an uncertain number of classes, while the positive can be clustered into one class. The detail of the algorithm is described in [7].

\section{GROUP BIASED DISCRIMINANT ANALYSIS}

Our Group Biased Discriminant Analysis (GBDA) extends BDA to handle multiple positive classes as discussed in Section 1 . Here, the problems are extended to $(x+y)$-class (multiple positive classes and multiple negative classes) problem. This type of query has not been studied since the traditional user interfaces did not allow the users to separate positive examples into multiple classes. With ImageGrouper, it is easily achieved. GBDA is characterized by following objective function,

$$
\mathbf{W}=\underset{\mathbf{W}}{\operatorname{argmax}}\left|\frac{\mathbf{W}^{\mathrm{T}} \mathbf{S}_{\mathrm{PN}} \mathbf{W}}{\mathbf{W}^{\mathrm{T}} \mathbf{S}_{\mathrm{W}} \mathbf{W}}\right|
$$

where $\mathbf{S}_{\mathrm{W}}$ is the sum of the within-class scatter matrix [1] of the positive groups defined as follows,

$$
\begin{gathered}
\mathbf{S}_{\mathrm{W}}=\sum_{\mathrm{i}=1}^{\mathrm{c}} \mathbf{S}_{\mathrm{i}} \\
\mathbf{S}_{\mathrm{i}}=\sum_{\mathrm{x} \in \mathrm{C}_{\mathrm{i}}}\left(\mathbf{x}-\mathbf{m}_{\mathrm{i}}\right)\left(\mathbf{x}-\mathbf{m}_{\mathrm{i}}\right)^{\mathrm{T}}
\end{gathered}
$$

$\mathbf{m}_{\mathrm{i}}$ is the mean vector of $i$-th positive class $\mathrm{C}_{\mathrm{i}}, c$ is the number of positive groups. $\mathbf{S}_{\mathrm{PN}}$ is the positive-to-negative scatter, which is introduced in this paper as follows,

$$
\begin{gathered}
\mathbf{S}_{\mathrm{PN}}=\sum_{\mathrm{i}=1}^{\mathrm{c}} \mathbf{S}_{\mathrm{Ni}} \\
\mathbf{S}_{\mathrm{Ni}}=\sum_{\mathrm{y} \in \mathrm{D}}\left(\mathbf{y}-\mathbf{m}_{\mathrm{i}}\right)\left(\mathbf{y}-\mathbf{m}_{\mathrm{i}}\right)^{\mathrm{T}}
\end{gathered}
$$

where, $\mathrm{D}$ is a set of negative examples.

In short, the GBDA tries to cluster each positive class while scatter the negative example away from the positive classes. Like BDA, each negative belongs to its own class. As in FDA [1], $\boldsymbol{W}$ is solved as the generalized eigenvector(s) associated with the largest eigenvalue(s) $\lambda$,

$$
\mathbf{S}_{\mathrm{PN}} \mathbf{w}_{\mathrm{i}}=\lambda_{\mathrm{i}} \mathbf{S}_{\mathrm{W}} \mathbf{w}_{\mathrm{i}}
$$

Finally, our discriminating transformation matrix [1] becomes,

$$
\boldsymbol{A}=\Phi \Lambda^{1 / 2}
$$

$\Phi$ is the matrix whose columns are the eigenvectors, and $\Lambda$ is the diagonal matrix of the corresponding eigenvalues. Once the transformation matrix is available, the distance between two images $\boldsymbol{x}$ and $\boldsymbol{y}$ is computed as follows.

$$
\operatorname{distance}(\mathbf{x}, \mathbf{y})=(\mathbf{x}-\mathbf{y})^{\mathrm{T}} \mathbf{A}(\mathbf{x}-\mathbf{y})
$$

In our current implementation, the calculates the distance between images in the database and the mean of each positive group. Then, the database images are ordered according to those distances. For more detail, see [4][7].

\section{ANALYSIS ON TOY PROBLEMS}

To Illustrate the effects of GBDA, we use three dimensional toy problems as shown in Figure 2. (a) and (b). The original data are plotted in 3D where positive examples are 'o's and negative ones are represented by ' $x$ 's. The projections of the data into two dimensional space are shown directly below. In this paper, we address two typical cases. The first case is when two positive clusters are distant from each other (Figure 2. (a)) The second case is when two positive clusters are close to each other (Figure 2. (b)) In both cases, negative examples are scattered across the problem space. We compared the results with Multiple Discriminant Analysis (MDA) [1] and BDA. In MDA, the negative examples are considered as one class. Thus, it deal with the problem as a three-class (two positive and one negative.)

In the first case, BDA merges two positive clusters into one cluster while MDA and GBDA preserve the separation of the two different clusters. GBDA clusters each positive class tighter than MDA. For the second case, GBDA and 

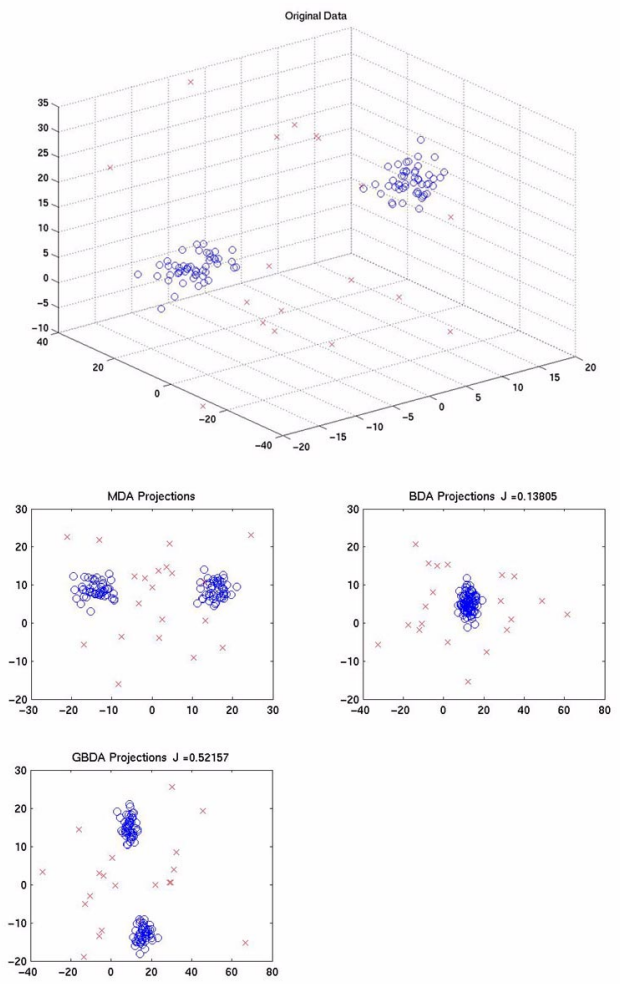

(a) Toy problem 1. GBDA performs similar to MDA.
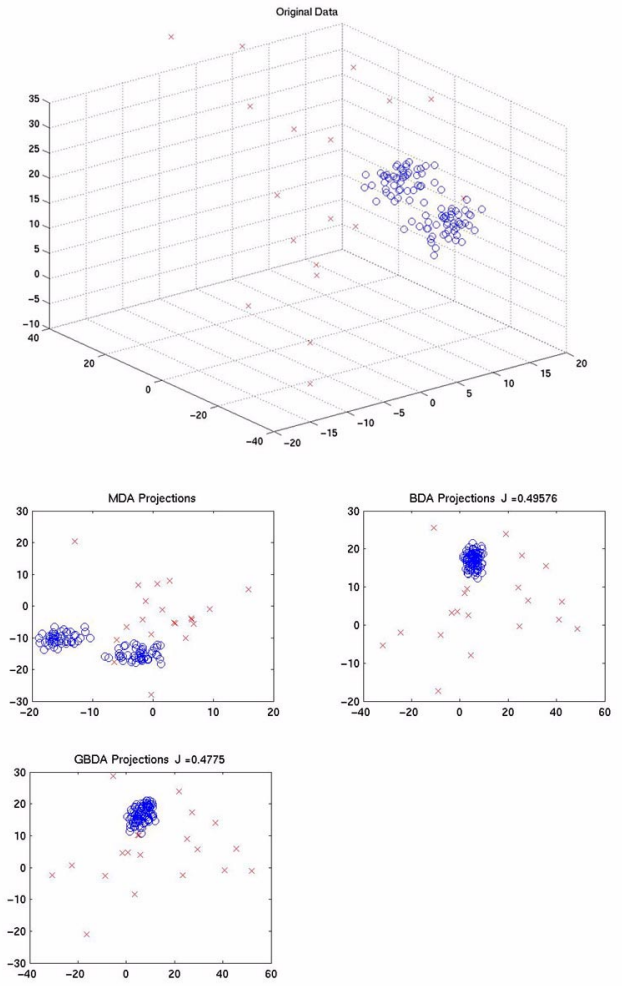

(b) Toy problem 2. GBDA performs similarly to BDA.

Figure 2. Toy problems (in 3D) and the result projections (2D.).
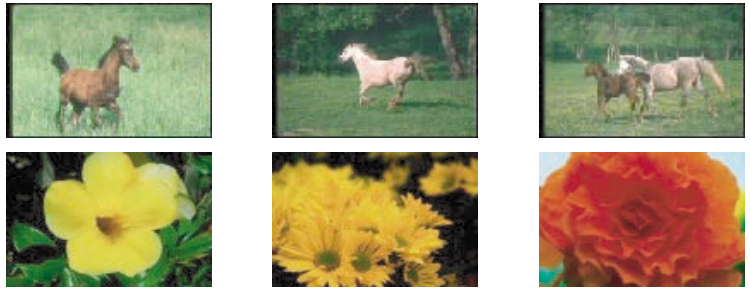

Figure 3. Examples of test images

BDA have similar effects: the two positive clusters are merged into one positive cluster. This is desirable effect since these two positive groups are very close to each other and should be considered as one group even though the user specifies them as different. Meanwhile, MDA keeps them separated. It also attracts negative examples near one of positive cluster. This effect is not suitable for image retrieval since it increases the possibility that many irrelevant images are retrieved.

\section{EXPERIMENTS ON REAL DATA}

In this section, we evaluate the performance of GBDA with real image retrieval problems.

\subsection{Data Sets}

To examine the advantages of GBDA over BDA, the retrieval performances of the two systems are tested on COREL image database. The dimensionality of the image feature space (color, texture and structure) is 37 [7]. We used a subset of 804 images from the database. These subset images consist of six classes of images: airplanes, cars, flowers, stained-glasses, horses and eagles. From each class, up to 40 images are given as positive query examples. For GBDA, the query examples are further divided into two sub-groups by the domain expert. For example, images of horses are divided into two subsets: "white horse" and "brown horses." For BDA, they are considered as one group. For simplicity, the sizes of the sub-groups are kept the same (up to 20.) Figure 3. shows some images of two representative test sets. For each test, equal number of images are picked from each sub-groups. Therefore, the total sample size ranges from 2 to 40 (in increments of two.)

\subsection{Performance Measures}

As a performance measure, we introduced weighted hit count. The weighted hit count is calculated as follows. For each image $\mathbf{x}_{\mathrm{i}}$ in the top 20 results,

$$
\text { whc }=\sum_{\mathrm{i}=1}^{20} \operatorname{hc}\left(\mathbf{x}_{\mathrm{i}}\right)
$$

where,

$$
h c\left(\mathbf{x}_{\mathrm{i}}\right)=\left\{\begin{array}{cl}
1 & \left(\boldsymbol{x}_{\mathrm{i}} \in \mathrm{C}\right) \\
-1 / \mathrm{i} & \text { (otherwise) }
\end{array}\right.
$$

$\mathrm{C}$ is the class of target images. In this scheme, every relevance image in the result set is given one. Meanwhile, any false hits are given negative points based on their ranks 


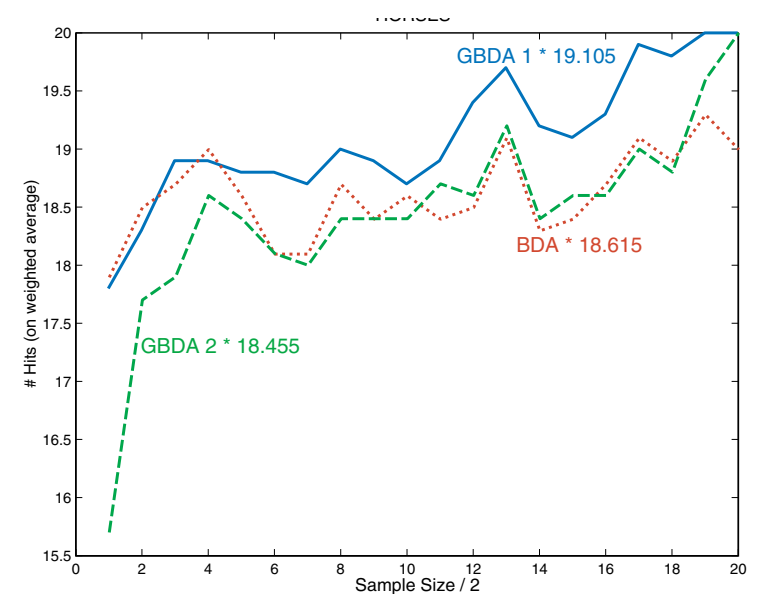

(a) Horse Example

Figure 4. Comparison of BDA and GBDA

in the result set. Therefore, false hits that are closer to the top of the list are penalized more than those at the bottom of the list. This measure reflects the quality of returned ranking as well as the number of correctly returned images. The graph shows the averages of 10 tests.

\subsection{Results}

As in the toy problems, we describe the results of two typical cases where GBDA outperforms BDA and where GBDA performs similarly to BDA. Figure 4. (a) and (b) shows the result of "horses" and "flowers" respectively. The horizontal axis is the number of positive examples. The vertical axis is the weighted hit count described above. GBDA-1 returns the top 10 images from each group. On the other hand, GBDA-2, order the images by the closer of the distances to the two groups and the top 20 images are returned.

In the case of "horses" (Figure 4. (a)), when the number of query examples is small (sample size $<8$ ), GBDA-1 shows the similar performances as BDA. GBDA-2 performs worse than BDA. When the number of samples gets larger (sample size > 8), GBDA-1 performs better than BDA, while GBDA-2 shows similar performance as BDA. This is corresponds to the first case of the toy problems. It also shows that in order for GBDA to model the group information correctly, it requires several image samples for each group.

Figure 4. (b) shows the results of "red and yellow flower" images. In this case, there are no significant differences between BDA, GBDA-1 and GBDA-2. This is because two groups are too close to each other for GBDA to create two separated clusters. Note that GBDA does not do anything harmful even if it cannot utilize group information. In this situation, it has similar effects as BDA. This corresponds to the second case of the toy problems.

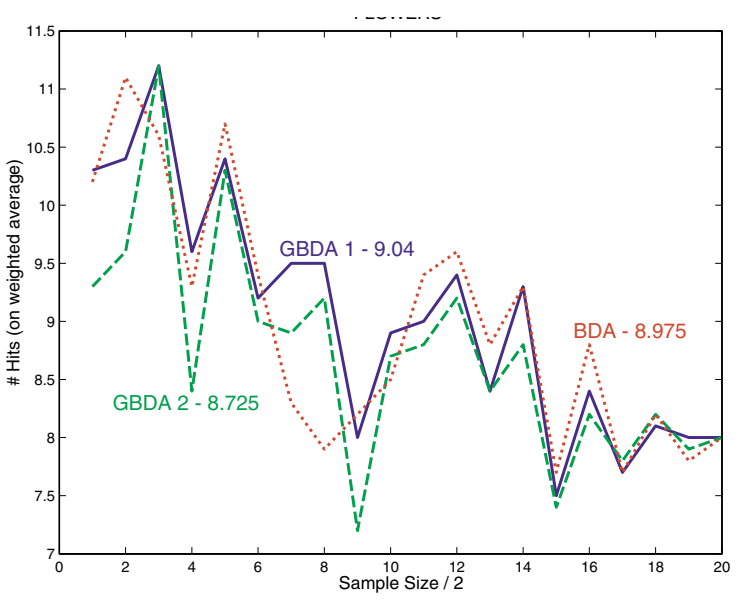

(b) Flower Example

\section{DISCUSSION AND CONCLUSION}

In this paper, we evaluated GBDA algorithm on toy problems of synthesized data as well as real image retrieval problems. Our experiment shows that GBDA separates the positive clusters when they are different enough and merges them when they are too close to separate. This is a desirable feature since in the Content-based Image Retrieval, it is very difficult for the user to judge the similarity in terms of low-level visual features. Thus, even if the user separates positive images into two groups, these two may not be distinct enough in the feature space. In this case, GBDA automatically merges them into one group while keeping it away from negative examples.

\section{ACKNOWLEDGEMENTS}

This work was supported in part by National Science Foundation Grant CDA 96-24396.

\section{REFERENCES}

[1] Duda, R.O., Hart, P.E. and D.G. Stork, Pattern Classification (2nd Ed.), Wiley-Interscience, 2000.

[2] Flickner, M. et al., "Query by image and video content: The QBIC system,” IEEE Computers, 1995.

[3] Nakazato, M., Manola, L. and Huang, T.S., "ImageGrouper: Search, Annotate and Organize Images by Groups," In Proc. of 5th Intl. Conf. on Visual Information Systems (VISual'02), 2002.

[4] Nakazato, M. and Huang, T. S., "Extending Image Retrieval with Group-Oriented Interface," In IEEE Intl' Conf. on Multimedia and Expo (ICME 2002), 2002.

[5] Rui, Y., et al., "Relevance Feedback: A Power Tool for Interactive Content-Based Image Retrieval," In IEEE Trans. on Circuits and Video Technology, Vol.8(5), 1998.

[6] Rui, Y. and Huang, T. S., "Optimizing Learning in Image Retrieval," In Proc. of IEEE CVPR, 2000.

[7] Zhou, X. S., et al. Comparing Discriminating Transformations and SVM for Learning during Multimedia Retrieval. In Proc. of ACM Multimedia '01, 2001. 